\title{
Nilotinib combined with interleukin-2 mediates antitumor and immunological effects in a B16 melanoma model
}

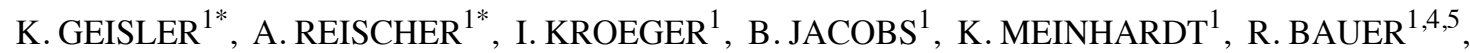 \\ B. RYFFEL ${ }^{2,3}$, A. MACKENSEN ${ }^{1}$ and E. ULLRICH ${ }^{1,4,5}$ \\ ${ }^{1}$ Department of Internal Medicine 5, Hematology/Oncology, University of Erlangen-Nürnberg, Erlangen, Germany; \\ ${ }^{2}$ INEM UMR 7355, CNRS and University Orléans, France; ${ }^{3}$ IIDMM, University of Cape Town, \\ Cape Town, South Africa; ${ }^{4}$ Laboratory of Cellular Immunology, Children's Hospital, \\ Department of Pediatric Hematology and Oncology, Goethe University, Frankfurt; \\ ${ }^{5}$ Center for Cell and Gene Therapy, Goethe University, Frankfurt, Germany
}

Received August 22, 2013; Accepted September 23, 2013

DOI: 10.3892/or.2014.3070

\begin{abstract}
The immune system contributes to tumor cell killing which can be enhanced by cancer chemotherapeutics and immune modulatory pharmaceuticals such as tyrosine kinase inhibitors (TKIs). Recently, the beneficial effect of natural killer $(\mathrm{NK})$ cells was demonstrated when combining interleukin-2 (IL-2) with the TKI imatinib. The aim of the present study was to address the antitumor and immunological effects of recently approved TKIs. Therefore, we focused on the comparison of the efficacy between imatinib and nilotinib in combination with IL-2 in a murine B16F10 melanoma model. Both TKIs possessed antitumor activity in vivo. However, the combination of nilotinib and IL-2 showed a superior outcome. Importantly, both the use of immunodeficient Rag $2 \gamma \mathrm{c}^{-/-}$mice, which lack T-lymphocytes, B-lymphocytes and NK cells, as well as NK cell-depletion in $\mathrm{C} 57 \mathrm{Bl} / 6$ mice reduced the therapeutic effect of nilotinib. Flow cytometry revealed a significant increase in the IFN- $\gamma$-producing $\mathrm{CD} 27^{+} \mathrm{NK}$ cell subpopulation following treatment with nilotinib and IL-2. Furthermore, the therapeutic antitumor effect of nilotinib/IL-2 was completely lost in IFN- $\gamma^{-/}$mice. In summary, we suggest that nilotinib
\end{abstract}

Correspondence to: Dr E. Ullrich, Laboratory of Cellular Immunology, Children's Hospital, Department of Pediatric Hematology and Oncology, Goethe University, Theodor-Stern-Kai 7, D-60590 Frankfurt, Germany

E-mail: evelyn.ullrich@kgu.de

*Contributed equally

Abbreviations: CML, chronic myeloid leukemia; Dasa, dasatinib; DC, dendritic cell; FACS, fluorescence-activated cell sorting; IFN, interferon; IL, interleukin; IMA, imatinib; Nilo, nilotinib; NK cell, natural killer cell; $\mathrm{O} / \mathrm{N}$, over night; TKI, tyrosine kinase inhibitor

Key words: natural killer cell, tyrosine kinase inhibitor, nilotinib, interleukin-2, tumor immunology combined with IL-2 confers high antitumor activity involving the subset of IFN- $\gamma$-producing $\mathrm{CD} 27^{+} \mathrm{NK}$ cells. These new insights are of high importance for the understanding and development of immunotherapeutic protocols using TKIs.

\section{Introduction}

Tyrosine kinase inhibitors (TKIs) have a great impact on routine treatment in oncology. Imatinib (STI-571, Glivec ${ }^{\circledR}$, Gleevec ${ }^{\circledR}$ ), the prototype of TKIs, was approved in 2003 for the first-line therapy of chronic myeloid leukemia (CML) (1). Since 2010, various TKIs, such as nilotinib (Tasigna ${ }^{\circledR}$ ), have been developed for the first-line therapy of CML patients $(2,3)$. Apart from CML, TKIs such as imatinib have been approved for firstline therapy of gastrointestinal stromal tumors (GIST), renal cell carcinoma (RCC), melanoma or a subgroup of non-small cell lung cancers (NSCLCs) (4-6). In CML, imatinib targets the BCR/ABL-fusion protein known as the central oncogenic signaling cascade resulting in a reduction of leukemic cells without additional chemotherapy (7). Nevertheless, some patients are or become resistant to imatinib. Therefore, new BCR/ABL-inhibitors, such as nilotinib and dasatinib, have been developed to overcome imatinib resistance $(2,3)$. Apart from its direct cell-autonomous effect, additional immunostimulation, mediated by host cells such as dendritic cells (DCs) and natural killer (NK) cells, was demonstrated in a murine B16F10 melanoma model (8). NK cells play a central role in antitumor immunity. They recognize virus-infected or transformed tumor cells resulting in direct cytotoxic killing of these targets and cytokine secretion consequently inducing a general immune response (9). NK cells are defined as CD3-negative, NKp46 and NK1.1-positive lymphocytes in $\mathrm{C} 57 \mathrm{Bl} / 6$ mice. They can be further subdivided into three groups by their surface expression of CD27 and CD11b (10).

In the present study, we addressed the role of nilotinib, as a TKI with a different selectivity profile to that of imatinib, in combination with IL-2 in a B16 melanoma model (11). Our results clearly demonstrated an elevated antitumor effect of nilotinib/IL-2 when compared to that of imatinib/IL-2. 
Notably, the therapeutic effect of nilotinib/IL-2 was lost in immunodeficient mice and following depletion of NK cells. The increase in $\mathrm{CD} 27^{+} \mathrm{NK}$ cells as the main source of IFN- $\gamma$ among NK cells as well as the abolished antitumor efficacy of the treatment protocol in IFN- $\gamma^{-/-}$mice emphasize the specific importance of the subset of IFN- $\gamma$-producing CD27+ NK cells. These new findings provide new insights to further improve immunotherapeutic protocols.

\section{Materials and methods}

Animals. Female C57Bl/6 mice were obtained from Elevage Janvier (Le Genest St. Isle, France), were housed in the Franz Penzoldt Centre (University Erlangen, Germany) and were used at 7-9 weeks of age. Rag $2 \gamma \mathrm{c}^{-/-}$mice and IFN- $\gamma$ knockout mice were kindly provided by Falk Nimmerjahn (Erlangen, Germany) and Bernard Ryffel (Orléans, France), respectively. Animal experiments were approved by the Regierung of Mittelfranken and Hessen, Germany.

Flow cytometry. Single-cell suspensions were stained with FITC-or PB-conjugated anti-CD11b(M1/70),PerCP-conjugated anti-CD3ع (145-2C11), PerCP-conjugated anti-CD19 (1D3), PE-conjugated anti-CD27 (LG.3A11), PE-Cy7-conjugated antiNK1.1 (PK136) and APC- and V450-conjugated anti-NKp46. Antibodies were purchased from BioLegend (San Diego, CA, USA), BD Biosciences (Heidelberg, Germany) and Miltenyi (Bergisch Gladbach, Germany). FACS experiments were performed on a FACSCanto II instrument (BD Biosciences) and analyzed by FlowJo software (Tree Star, Ashland, OR, USA).

Functional analysis. IFN- $\gamma$ production was assessed by ELISA after $\mathrm{O} / \mathrm{N}$ co-incubation with B16 cells (at an effector-target ratio of 10:1) and 5,000 U/ml IL-2. ELISA was performed as described by the supplier (BD Biosciences). The tumor lysis capacity of B16 melanoma cells was investigated by crystal violet assay as previously described (12).

Melanoma model and preparation of TKI solutions. The B16 melanoma model was established as previously described (13). Briefly, 500,000 B16F10 cells were injected i.v. on day 0, and mice received an oral application of TKIs twice daily (b.i.d.) until day 11 as well as an intraperitoneal (i.p.) injection of 100,000 U IL-2 (b.i.d.) from day 6 until the end of the experiment. Imatinib (Novartis, Basel, Switzerland) was used at a daily dosage of 75 or $150 \mathrm{mg} / \mathrm{kg}$ body weight diluted in $100 \mu 1$ polyethylene glycol (PEG) 300 (Sigma-Aldrich, Steinheim, Germany). Nilotinib (Novartis) was first diluted in 1-methyl-2-pyrrolidinone solution at $0.2 \%$ and afterwards was administered at a daily dose of 37.5 or $75 \mathrm{mg} / \mathrm{kg}$ body weight diluted in $100 \mu 1$ PEG 300. Dasatinib (Bristol-Myers Squibb, New York, NY, USA) was used at a daily dosage of $25 \mathrm{mg} / \mathrm{kg}$ body weight diluted in $100 \mu 1$ PEG 300. Untreated controls received $100 \mu$ l PEG 300 p.o. and $100 \mu$ l PBS i.p.

\section{Results}

Nilotinib reduces the number of lung metastases in the B16 melanoma model. C57Bl/6 mice developing B16 melanoma metastases were treated with either nilotinib alone $(75 \mathrm{mg} / \mathrm{kg}$ per day) or in combination with IL-2 (100,000 U), and the number of metastases were compared to an untreated control group. Following analysis of the number of lung metastases in the tumor-bearing mice, a significant reduction in number was noted in the groups treated with nilotinib alone or in combination with IL-2 (Fig. 1A). Yet, the combination of nilotinib and IL-2 led to the most impressive antitumor effect. As it was previously demonstrated that imatinib combined with IL-2 induces a superior tumor response than either substance alone $(8,14)$, we performed experiments directly comparing imatinib/IL-2 and nilotinib/IL-2. Our results revealed a superior effect in mice treated with nilotinib and IL-2 (Fig. 1B). The reduced number of lung metastases is further illustrated by images showing the lung of one representative animal per group (Fig. 1). Notably, a dose escalation of imatinib up to $300 \mathrm{mg} / \mathrm{kg}$ daily or nilotinib up to $150 \mathrm{mg} / \mathrm{kg}$ daily did not further improve the antitumor potency (data not shown). In an additional experiment, we tested dasatinib as a multi-targeted TKI also approved for first-line therapy of CML $(2,15)$. When directly comparing dasatinib and nilotinib as a treatment option in the murine melanoma model, both in combination with IL-2, dasatinib did not demonstrate an evident reduction in lung metastases when compared to that observed following treatment with nilotinib (Fig. 2). Furthermore, treatment with dasatinib was less well-tolerated when compared to the other TKIs as mice developed an effusion syndrome during dasatinib treatment. Based on the marked antitumor potency of nilotinib/IL-2, we aimed to further investigate the involvement of the immune system in this therapeutic concept.

The therapeutic effect of nilotinib combined with $I L-2$ is NK cell-dependent. To assess the impact of the immune system on the positive antitumor effects of nilotinib/IL-2, we injected B16 melanoma cells into Rag $2 \gamma^{-/-}$mice lacking T-lymphocytes, B-lymphocytes and NK cells. In these immunodeficient mice we observed at least a 2.5 -fold higher number of lung metastases when compared to this number in the $\mathrm{C} 57 \mathrm{Bl} / 6$ wild-type (WT) mice. Moreover, the therapeutic effect of nilotinib/IL-2 was completely abrogated in the Rag $2 \gamma \mathrm{c}^{-/-}$mice, revealing a possible role for T-lymphocytes, B-lymphocytes and NK cells in the antitumor potency (Fig. 3A). Next, we analyzed the impact of NK cells in this model. By using anti-NK1.1-specific antibodies, we effectively depleted NK cells in C57Bl/6 mice. In general, the mice depleted of NK cells had a significantly higher number of lung metastases. Again, the therapeutic effect of the nilotinib/IL-2 combination therapy was nullified in the treated group (Fig. 3B). These data are illustrated by images of the metastasis-bearing lungs (Fig. 3B, below the graph).

Nilotinib/IL-2 treatment increases the number of $C D 27^{+}$ IFN- $\gamma$-producing $N K$ cells. We next performed extensive immune monitoring by flow cytometry focusing on NK cell subpopulations in different organs of the tumor-bearing mice that were either treated or not with nilotinib/IL-2. The gating strategy to assess NK cell subsets distinguished by the expression of CD27 and CD11b is shown in Fig. 4A. Regarding the distribution of different NK cell subsets in peripheral organs, a significant increase in $\mathrm{CD}_{2} 7^{+} \mathrm{CD} 11 \mathrm{~b}-\mathrm{NK}$ cells was observed in the lung and spleen of the nilotinib/IL-2-treated mice when 

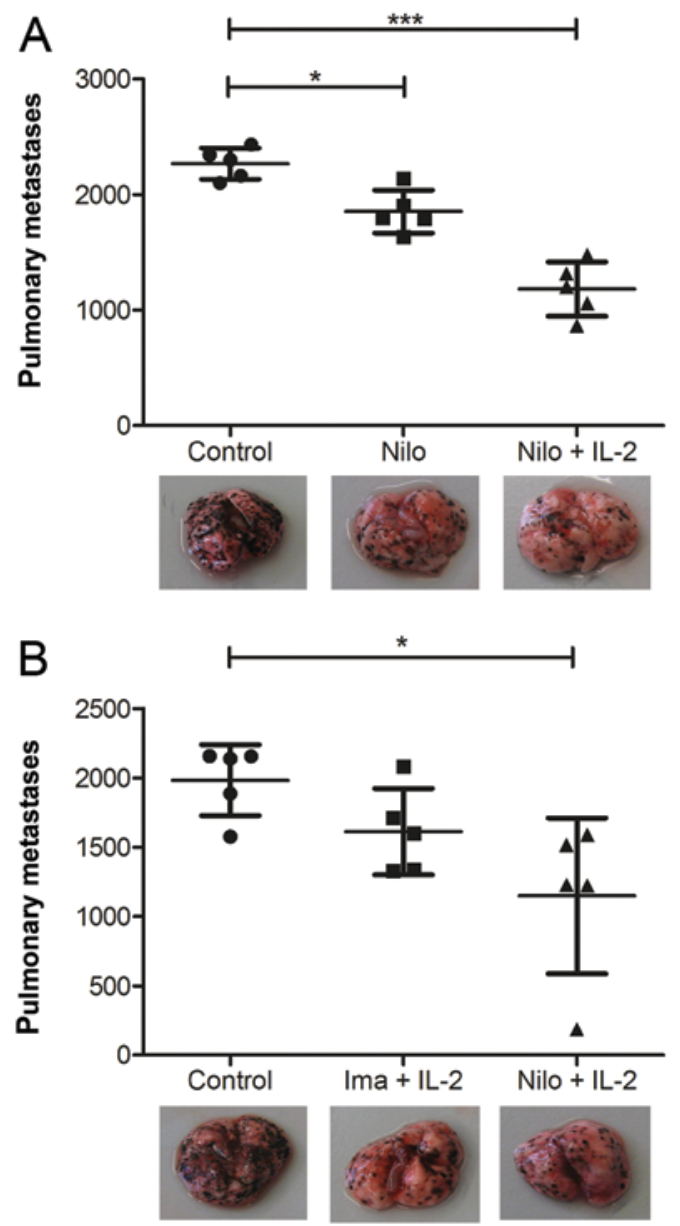

Figure 1. Number of pulmonary metastases following treatment with nilotinib or imatinib both combined with IL-2. C57Bl/6 mice received an i.v. tumor injection on day 0 with 500,000 B16F10 melanoma cells and were treated for 11 days with TKI p.o. and from day 7 on with IL-2 i.p. (A) Treatment with nilotinib (Nilo, $75 \mathrm{mg} / \mathrm{kg}$ daily) alone or in combination with IL-2 (100,000 U b.i.d.).(B) Treatment with imatinib (Ima, $150 \mathrm{mg} / \mathrm{kg}$ daily) combined with IL-2 (100,000 U b.i.d.) or Nilo (75 mg/kg daily) combined with IL-2 (100,000 U b.i.d.). (A and B) The vehicle for the medication was PEG 300 and was also administered to the control group. The graphs show one representative experiment with $\mathrm{n}=5$ mice/group. Below each graph, one image of a representative metastatic lung is depicted/group. One-way ANOVA was used for statistical evaluation. ${ }^{*} \mathrm{P} \leq 0.5,{ }^{* * *} \mathrm{P} \leq 0.001$.

compared to that in the untreated controls (Fig. 4B and C). In contrast, in the same organs, a significant reduction in $\mathrm{CD} 27$ $\mathrm{CD}_{11 \mathrm{~b}}{ }^{+} \mathrm{NK}$ cells was noted in the treated mice. A significant increase in the $\mathrm{CD} 27^{+} \mathrm{CD} 11 \mathrm{~b}^{+}$intermediate $\mathrm{NK}$ cell subset was only found in the lung of the treated mice but not in the spleen. We further addressed the functional relevance of sorted $\mathrm{CD} 27^{+} \mathrm{NK}$ cells in comparison to $\mathrm{CD} 11 \mathrm{~b}^{+} \mathrm{NK}$ cells in vitro. Importantly, co-cultivation of purified NK cell subpopulations with B16 melanoma cells led to a significantly higher IFN- $\gamma$ secretion of the $\mathrm{CD} 27^{+} \mathrm{NK}$ cells when compared to that of the CD11b ${ }^{+}$NK cell subpopulation (Fig. 4D).

IFN- $\gamma$ is suggested as a key player in the antitumor effect mediated by nilotinib/IL-2. Based on the knowledge that the number of IFN- $\gamma$-secreting NK cells are increased during therapy with nilotinib/IL-2, we aimed to ascertain whether IFN- $\gamma$ is relevant for the outcome of this therapeutic regimen. To solve this issue, we used IFN- $\gamma$ knockout mice with a

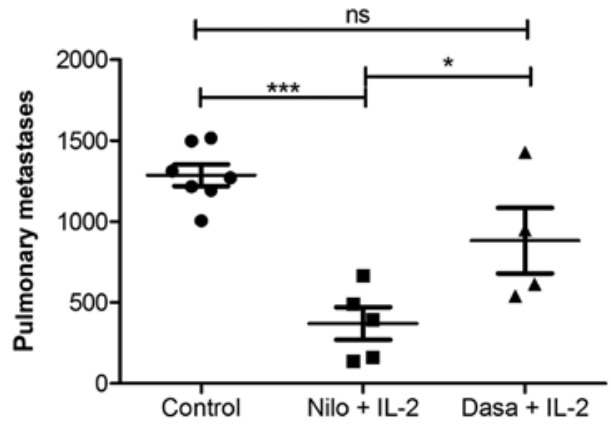

Figure 2. Effects of treatment with dasatinib/IL-2 on pulmonary metastases. The number of pulmonary metastases following treatment with nilotinib (Nilo) or dasatinib (Dasa), each combined with IL-2. C57Bl/6 mice received 500,000 B16 melanoma cells on day 0 and were treated for 12 days with either Nilo $75 \mathrm{mg} / \mathrm{kg}$ or Dasa $25 \mathrm{mg} / \mathrm{kg}$, each combined with IL-2 (100,000 U b.i.d.). The control group was treated with PEG 300. The graph shows one representative experiment with $n=4-6$ mice/group. One-way ANOVA was used for statistical evaluation. ${ }^{*} \mathrm{P} \leq 0.5,{ }^{* * * *} \mathrm{P} \leq 0.001$. ns, not significant.

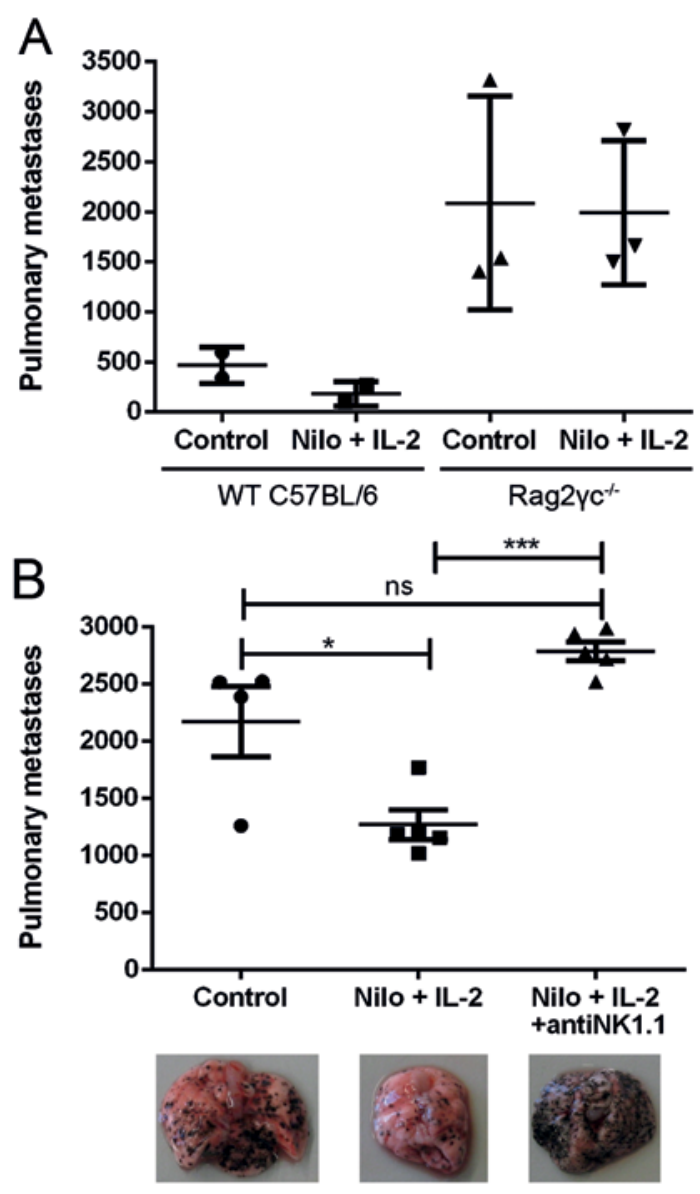

Figure 3. Number of pulmonary metastases after treatment with nilotinib combined with IL-2 in immunodeficient mice. (A) C57Bl/6 wild-type (WT) mice and $\mathrm{Rag} 2 \gamma \mathrm{c}^{-/}$mice received 500,000 B16F10 melanoma cells i.v. and were treated for 7 days with nilotinib (Nilo, $75 \mathrm{mg} / \mathrm{kg}$ ) and IL-2 (100,000 U b.i.d.). The control group was treated with PEG 300. The number of lung metastases are indicated from one representative experiment of 3 with $\mathrm{n}=2-5$ mice/group. (B) C57Bl/6 mice received 500,000 B16F10 cells on day 0 and were treated for 10 days with Nilo $(75 \mathrm{mg} / \mathrm{kg})$ and IL-2 (100,000 U b.i.d.). NK cell depletion was performed by i.p. injection of anti-NK1.1 antibody on day $-4,0$ and +4 . The control group was treated with PEG 300. The number of lung metastases in one representative experiment with $n=3-5$ mice/group is shown. Image of one representative lung per group is depicted for visualization of the metastatic disease. (A and B) One-way ANOVA was used for statistical evaluation. ${ }^{*} \mathrm{P} \leq 0.5,{ }^{* * *} \mathrm{P} \leq 0.001$. ns, not significant. 
A
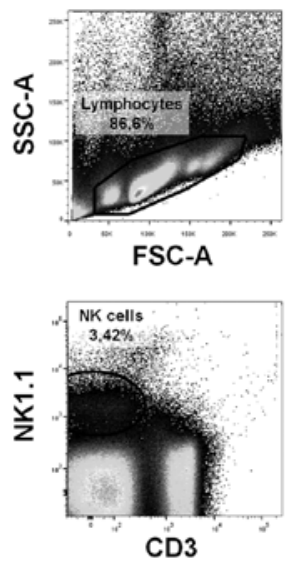

B $\mathrm{CD}^{2} 7^{\circ} \mathrm{CD} 11 \mathrm{~b} \cdot \mathrm{NK}$ cells in spleen
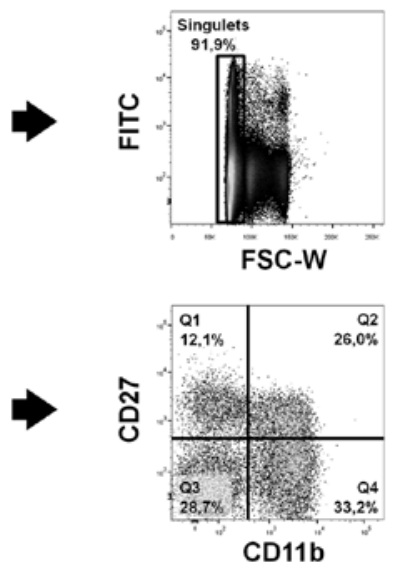

$\mathrm{CD} 27^{*} \mathrm{CD} 11 \mathrm{~b}+\mathrm{NK}$ cells in spleen
D

INF $\gamma$ production of $\mathrm{CD} 27^{+} \mathrm{NK}$ cells

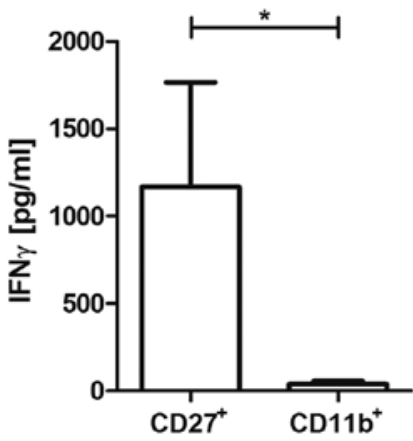

CD27-CD11b+NK cells in spleen
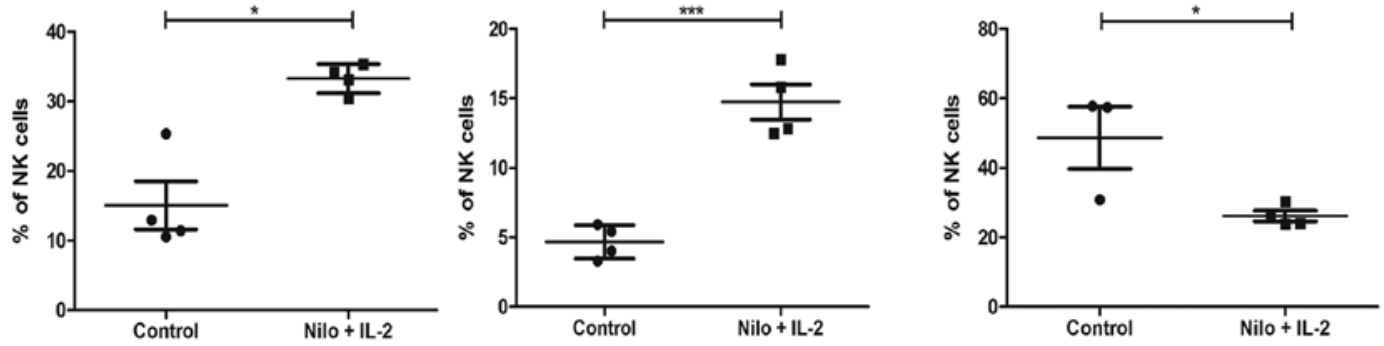

C $\quad$ D27 $7^{+}$D $11 b-$ NK cells in lung

$\mathrm{CD} 27^{+} \mathrm{CD} 11 \mathrm{~b}^{+} \mathrm{NK}$ cells in lung

CD27 CD11 $\mathrm{b}^{+} \mathrm{NK}$ cells in lung
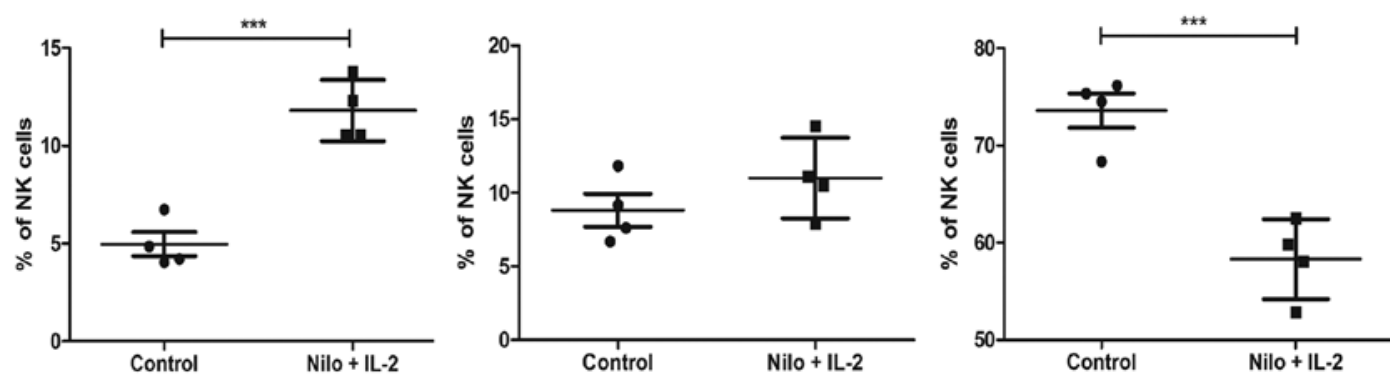

Figure 4. Phenotypical and functional analysis of the subpopulations of NK cells following treatment with nilotinib/IL-2. (A) Flow cytometry gating strategy of NK subsets using anti-NK1.1, anti-CD3, anti-CD27 and anti-CD11b as described in Materials and methods. (B and C) C57BL/6 mice were injected with 500,000 B16F10 melanoma cells on day 0 and treated with nilotinib (Nilo, $75 \mathrm{mg} / \mathrm{kg}$ ) combined with IL-2 (100,000 U b.i.d.). The control group was treated with PEG 300. After 10 days, cells from the spleen (B) and lung (C) were analyzed by FACS as described in Materials and methods. One-way ANOVA was used for statistical evaluation. ${ }^{*} \mathrm{P} \leq 0.5,{ }^{* * *} \mathrm{P} \leq 0.001$. (D) IFN- $\gamma$ secretion was measured by ELISA in the supernatant from sorted CD27 $7^{+}$or CD11b ${ }^{+} \mathrm{NK}$ cells after $\mathrm{O} / \mathrm{N}$ stimulation with B16 melanoma cells and 5,000 U/ml IL-2 in vitro. t-test was used for statistical evaluation; ${ }^{*} \mathrm{P} \leq 0.5$.

C57B1/6 background injected with B16 melanoma cells and used exactly the same therapeutic protocol as for WT C57Bl/6 mice. Indeed, in the IFN- $\gamma^{-/-}$mice, nilotinib combined with IL-2 completely lost its antitumor efficacy as shown by an equally high number of lung metastases. Images of one representative animal per group are shown in Fig. 5A. This result is in line with our observation of an elevated IFN- $\gamma$ concentration in supernatants of splenocytes from mice that were treated with nilotinib/IL-2 in vivo and further stimulated with B16 tumor cells in vitro (Fig. 5B). In summary, these experiments emphasize the role of IFN- $\gamma$ in the antitumor immune response induced by nilotinib/IL-2 which is possibly mediated by the subset of CD $27^{+} \mathrm{NK}$ cells.

\section{Discussion}

Since imatinib was clinically approved as the first TKI for the treatment of CML more than 10 years ago, TKIs are frequently used in daily clinical routine. During the last few years, two second-generation BCR/ABL TKIs, nilotinib and dasatinib, have been approved for the first-line therapy of $\operatorname{CML}(2,3)$. The combination of imatinib and IL-2 has been reported to interfere with the immune system, particularly with DC and NK cells $(8,14)$. Recently, a phase I clinical trial was carried out using a combination of imatinib plus IL-2 and low-dose cylcophosphamid in patients with refractory solid tumors. The level of HLA-DR ${ }^{+} \mathrm{NK}$ cells in the blood of the patients was positively correlated with progression-free survival (PFS) and overall survival (OS) (16).

In the present study, we investigated the effects on the immune system induced by nilotinib therapy. Using a B16F10 mouse melanoma model, we observed only a moderate reduction in the number of lung metastasis induced by nilotinib alone, possibly due to an impaired angiogenesis as a result of PDGFR inhibition. Interestingly, the combination of nilotinib (daily $75 \mathrm{mg} / \mathrm{kg}$ ) plus IL-2 significantly reduced the 
A

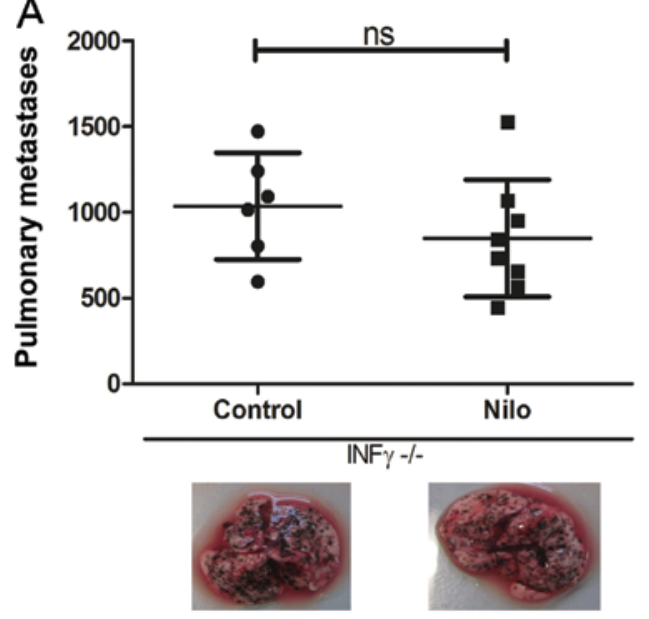

B

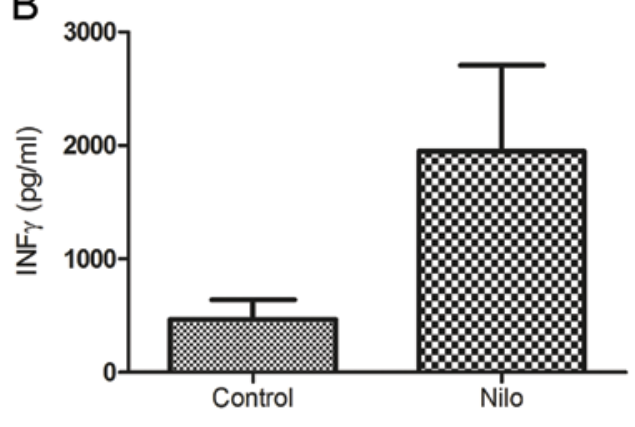

Figure 5. Pulmonary metastases after treatment with nilotinib/IL-2 in IFN- $\gamma^{-/}$mice. (A) C57BL/6 wild-type mice and IFN- $\gamma$ knockout mice were injected with 500,000 B16F10 melanoma cells i.v. and treated for 10 days with nilotinib (Nilo, $75 \mathrm{mg} / \mathrm{kg}$ ) and IL-2 (100,000 U b.i.d.). The control group was treated with PEG 300. The graph shows one representative experiment from 3 with $n=3-6$ mice/group. Images below depict one representative lung of both groups illustrating treatment failure. (B) Production of IFN- $\gamma$ after a $24-\mathrm{h}$ stimulation of splenocytes with IL-2 from either controls or mice treated with Nilo (75 mg/kg daily). (A and B) t-test was used for statistical evaluation. ns, not significant.

number of lung metastases when compared to the number in the untreated controls (Fig. 1). In our murine tumor model, escalating doses of nilotinib $\left(150 \mathrm{mg} / \mathrm{m}^{2}\right.$ daily) did not have any additional benefit. When comparing nilotinib/IL-2 with imatinib/IL-2, a clear advantage was observed for the therapeutic protocol using nilotinib. Importantly, our observation in the murine model is in line with clinical data from the international multi-center ENESTnd trial using nilotinib as first-line therapy for newly diagnosed $\mathrm{Ph}^{+} \mathrm{CML}$ in the chronic phase resulting in improved rates of major molecular response (MMR) at 12 months (43-44 vs. 22\%; $\mathrm{P}<0.001)$ and complete cytogenetic response rates (CCyR; 78-80 vs. 65\%; $\mathrm{P}<0.001)$ compared to standard treatment with imatinib (3). In addition, the DASISION trial for first-line treatment of CML patients revealed that dasatinib demonstrated better CCyR and MMR in comparison to imatinib (2). In contrast, in our murine tumor model, we did not observe a comparable positive effect by dasatinib treatment, even in combination with IL-2 (Fig. 2). Moreover, dasatinib-treated mice frequently developed pleural effusion and ascites as a side effect that may be in line with reports of pleural effusion by at least some patients included in the DASISION trial (2).

In preclinical studies, Salih et al (17) addressed the impact of imatinib, dasatinib and nilotinib on human NK cell function in vitro. Whereas imatinib did not have any effect on human NK cell function (cytotoxicity and cytokine production), nilotinib impaired NK cell cytokine production at a high dosage, and dasatinib abrogated both NK cell cytotoxicity as well as cytokine production, possibly by inducing apoptosis in the CD56 ${ }^{\text {high }}$ NK cell subset (17).

Due to the significant reduction in tumor progression following nilotinib/IL-2 treatment in the present study, we further focused on the impact of this therapeutic protocol on the immune system. It has been demonstrated that imatinib does not exclusively act by direct cytotoxicity on B16F10 tumor cells in vitro $(8,14)$, but the antitumor effect is mediated by additional stimulation of the immune system. In line with this finding, we observed a beneficial stimulating impact of nilotinib on the immune system. By using Rag $2 \gamma \mathrm{c}^{-/-}$ mice lacking T-lymphocytes, B-lymphocytes and NK cells, we demonstrated that the antitumor effect of nilotinib was dependent on immune cells (Fig. 3). In particular, NK cells played an important role as the antitumor effect observed by nilotinib/IL-2 was completely abrogated after depletion of NK cells (Fig. 3B). Concerning the distribution of the different NK cell subsets, we found an increase in the cytokine-producing $\mathrm{NK}$ cell subset $\left(\mathrm{CD} 27^{+} \mathrm{CD} 11 \mathrm{~b}^{-}\right)$in the lung and spleen of nilotinib/IL-2 treated mice and a decrease in mature $\mathrm{CD} 27^{-} \mathrm{CD}_{11 b^{+}} \mathrm{NK}$ cells in the same compartments (Fig. 4). We suggest that this distribution results from a higher production of more immature $\mathrm{CD} 27^{+} \mathrm{NK}$ cells. Importantly, the $\mathrm{CD} 27^{+} \mathrm{NK}$ cell subpopulation was determined to be the main producer of high amounts of IFN- $\gamma$.

Notably, in line with these observations, we measured high amounts of IFN- $\gamma$ in the supernatants of splenocytes isolated from mice that were treated with nitolinib (Fig. 5). The fact that Salih et al (17) demonstrated a negative impact of nilotinib on NK cell cytokine production could be explained by the elevated dosing when tested in vitro. In our model, oral application may have led to a more physiologically relevant plasma concentration of nilotinib. It is notable that Hassold et al (18) found that dasatinib inhibited NK cell function during functional assays but was even able to enhance cytokine secretion and cytotoxicity after a 24-h wash-out period of dasatinib.

We further confirmed the relevance for IFN- $\gamma$ in vivo by use of IFN- $\gamma^{-/-}$mice as tumor bearers. IFN- $\gamma^{-/-}$mice exhibited no antitumor efficacy upon treatment with nilotinib/IL-2 (Fig. 5).

In summary, we report a high antitumor potential of the combination therapy of nilotinib/IL-2. We further suggest a major role of IFN- $\gamma$-producing $\mathrm{CD} 27^{+} \mathrm{NK}$ cells in the therapeutic effects observed. The immune-stimulating effect of nilotinib/IL-2 was superior to that of imatinib/IL-2 and dasatinib/IL-2. This positive immune modulation may be an additional reason for the superiority of nilotinib when 
compared to imatinib in first-line therapy for CML apart from the direct inhibition of the BCR/ABL transcript.

\section{Acknowledgements}

The authors are grateful to Alexandra Abendroth, Julia Schneider and Franziska Ganss for their technical assistance and to PD Dr Jörg Distler and Professor Falk Nimmerjahn for material support and helpful discussions. This study was supported by Novartis, Bristol-Myers Squibb, and Bayer Health Care. The Laboratory of EU was supported by the German Cancer Aid (Max Eder Nachwuchsgruppe, Deutsche Krebshilfe), the Wilhelm Sander Foundation and the LOEWE Center for Cell and Gene Therapy, Frankfurt, funded by the Hessian Ministry of Higher Education, Research and the Arts, Germany (III L 4-518/17.004).

\section{References}

1. O'Brien SG, Guilhot F, Larson RA, et al: Imatinib compared with interferon and low-dose cytarabine for newly diagnosed chronic-phase chronic myeloid leukemia. N Engl J Med 348: 994-1004, 2003.

2. Kantarjian H, Shah NP, Hochhaus A, et al: Dasatinib versus imatinib in newly diagnosed chronic-phase chronic myeloid leukemia. N Engl J Med 362: 2260-2270, 2010.

3. Saglio G, Kim DW, Issaragrisil S, et al: Nilotinib versus imatinib for newly diagnosed chronic myeloid leukemia. N Engl J Med 362: 2251-2259, 2010.

4. Rosell R, Carcereny E, Gervais R, et al: Erlotinib versus standard chemotherapy as first-line treatment for European patients with advanced EGFR mutation-positive non-small-cell lung cancer (EURTAC): a multicentre, open-label, randomised phase 3 trial. Lancet Oncol 13: 239-246, 2012.

5. Escudier B, Szczylik C, Hutson TE, et al: Randomized phase II trial of first-line treatment with sorafenib versus interferon alfa-2a in patients with metastatic renal cell carcinoma. J Clin Oncol 27: 1280-1289, 2009.
6. Blanke CD, Rankin C, Demetri GD, et al: Phase III randomized, intergroup trial assessing imatinib mesylate at two dose levels in patients with unresectable or metastatic gastrointestinal stromal tumors expressing the kit receptor tyrosine kinase: S0033. J Clin Oncol 26: 626-632, 2008 .

7. Hochhaus A, O'Brien SG, Guilhot F, et al: Six-year follow-up of patients receiving imatinib for the first-line treatment of chronic myeloid leukemia. Leukemia 23: 1054-1061, 2009.

8. Borg C, Terme M, Taieb J, et al: Novel mode of action of c-kit tyrosine kinase inhibitors leading to NK cell-dependent antitumor effects. J Clin Invest 114: 379-388, 2004.

9. Geller MA and Miller JS: Use of allogeneic NK cells for cancer immunotherapy. Immunotherapy 3: 1445-1459, 2011.

10. Hayakawa Y and Smyth MJ: CD27 dissects mature NK cells into two subsets with distinct responsiveness and migratory capacity. J Immunol 176: 1517-1524, 2006.

11. Manley PW, Stiefl N, Cowan-Jacob SW, Kaufman S, Mestan J, Wartmann M, Wiesmann M, Woodman R and Gallagher N: Structural resemblances and comparisons of the relative pharmacological properties of imatinib and nilotinib. Bioorg Med Chem 18: 6977-6986, 2010.

12. Meinhardt K, Kroeger I, Abendroth A, Müller S, Mackensen A and Ullrich E: Influence of NK cell magnetic bead isolation methods on phenotype and function of murine NK cells. J Immunol Methods 378: 1-10, 2012.

13. Ullrich E, Bonmort M, Mignot G, et al: Trans-presentation of IL-15 dictates IFN-producing killer dendritic cells effector functions. J Immunol 180: 7887-7897, 2008.

14. Taieb J, Chaput N, Menard C, et al: A novel dendritic cell subset involved in tumor immunosurveillance. Nat Med 12: 214-219, 2006.

15. Hantschel O, Rix U and Superti-Furga G: Target spectrum of the BCR-ABL inhibitors imatinib, nilotinib and dasatinib. Leuk Lymphoma 49: 615-619, 2008.

16. Chaput N, Flament C, Locher C, et al: Phase I clinical trial combining imatinib mesylate and IL-2: HLA-DR ${ }^{+} \mathrm{NK}$ cell levels correlate with disease outcome. Oncoimmunology 2: e23080, 2013.

17. Salih J, Hilpert J, Placke T, et al: The BCR/ABL-inhibitors imatinib, nilotinib and dasatinib differentially affect NK cell reactivity. Int J Cancer 127: 2119-2128, 2010.

18. Hassold N, Seystahl K, Kempf K, et al: Enhancement of natural killer cell effector functions against selected lymphoma and leukemia cell lines by dasatinib. Int J Cancer 131: E916-E927, 2012. 